\title{
Can a supernova be located by its neutrinos?
}

\author{
J. F. Beacom* and P. Vogel ${ }^{\dagger}$ \\ Department of Physics, California Institute of Technology, Pasadena, California 91125
}

(Received 23 November 1998; published 7 July 1999)

\begin{abstract}
A future core-collapse supernova in our Galaxy will be detected by several neutrino detectors around the world. The neutrinos escape from the supernova core over several seconds from the time of collapse, unlike the electromagnetic radiation, emitted from the envelope, which is delayed by a time of the order of hours. In addition, the electromagnetic radiation can be obscured by dust in the intervening interstellar space. The question therefore arises whether a supernova can be located by its neutrinos alone. The early warning of a supernova and its location might allow greatly improved astronomical observations. The theme of the present work is a careful and realistic assessment of this question, taking into account the statistical significance of the various neutrino signals. Not surprisingly, neutrino-electron forward scattering leads to a good determination of the supernova direction, even in the presence of the large and nearly isotropic background from other reactions. Even with the most pessimistic background assumptions, SuperKamiokande (SK) and the Sudbury Neutrino Observatory (SNO) can restrict the supernova direction to be within circles of radius $5^{\circ}$ and $20^{\circ}$, respectively. Other reactions with more events but weaker angular dependence are much less useful for locating the supernova. Finally, there is the oft-discussed possibility of triangulation, i.e., determination of the supernova direction based on an arrival time delay between different detectors. Given the expected statistics we show that, contrary to previous estimates, this technique does not allow a good determination of the supernova direction. [S0556-2821(99)03214-2]
\end{abstract}

PACS number(s): 97.60.Bw, 13.10.+q, 25.30.Pt, 95.55.Vj

\section{INTRODUCTION}

There has been great interest recently in the question of whether or not a supernova can be located by its neutrinos. If so, this may offer an opportunity to give an early warning to the astronomical community, so that the supernova light curves can be observed from the earliest possible time. An international supernova early alert network has been formed for this purpose, and the details of its implementation $[1,2]$ were the subject of a recent workshop [3]. (The creation of such a network was also discussed in Ref. [4].) One of the primary motivations for such a network is to greatly reduce the false signal rate by demanding a coincidence between several different detectors, as detailed in Refs. [1,2]. The second motivation, to locate the supernova by the neutrino signal, is the topic of this paper.

An early-warning network is important because supernovas are rare, with the estimated core-collapse supernova rate in the Galaxy about 3 times per century [5]. The present neutrino detectors can easily observe a supernova anywhere in the Galaxy or its immediate companions (e.g., the Magellanic Clouds). Unfortunately, the present detectors do not have large enough volumes to observe a supernova in even the nearest galaxy (Andromeda, about $700 \mathrm{kpc}$ away).

Since decisions about how to implement this network are being made now, it is of current and necessary practical interest to make detailed calculations of what can realistically be done. In this paper we carefully examine the available techniques for locating the supernova by its neutrino signal. The problem has been discussed in general before (see Ref.

\footnotetext{
*Electronic address: beacom@citnp.caltech.edu

${ }^{\dagger}$ Electronic address: vogel@lamppost.caltech.edu
}

[6], for example). (See also the early discussions in Refs. [7-9].) Previous estimates, in particular regarding the triangulation problem $[1,2,6,10,11]$, were rather optimistic. In this paper, we make explicit some of the underlying assumptions in these calculations, and explain what the fundamental limitations on the precision are. Generally speaking, we find that this problem is more difficult than had been anticipated. The results below were first presented at the above workshop [12].

There are two types of techniques to locate a supernova by its neutrinos. The first class of techniques is based on angular distributions of the neutrino reaction products, which can be correlated with the neutrino direction. In this case, a single experiment can independently announce a direction and its error. The second method of supernova location is based on triangulation using two or more widely separated detectors. This technique would require significant and immediate data sharing among the different experiments. Our conclusion, that triangulation is at best very crude, has an important impact on the ongoing design decisions for the type of alert network and the degree of data sharing.

Let us note in passing that the supernova might be not only located on the sky, i.e., in two dimensions, but that its distance can be also reasonably estimated. The number of neutrino events $N$ is proportional to the binding energy release $E_{B}$ of the supernova and of course falls off as the distance $D$ squared:

$$
N=N_{0}\left(\frac{E_{B}}{3 \times 10^{53} \mathrm{ergs}}\right)\left(\frac{10 \mathrm{kpc}}{D}\right)^{2},
$$

where $N_{0}$ is the number of events at the canonical values of $E_{B}$ and $D$. The binding energy is thought to be $E_{B}=(3.0$ 
$\pm 1.5) \times 10^{53}$ ergs [13], i.e., a relative precision of $50 \%$. The total numbers of events from all reactions are $N_{0} \simeq 10^{4}$ for Super Kamiokande (SK) and $N_{0} \simeq 10^{3}$ for Sudbury Neutrino Observatory (SNO) (the expected signals in SK and SNO are discussed in Refs. $[14,15])$. Note that $N_{0}$ depends on the neutrino spectrum temperatures (which can be measured). The relative errors in the measured $N$ and the calculated $N_{0}$ are much smaller than the relative error in $E_{B}$. Then

$$
\frac{\delta D}{D} \simeq \frac{1}{2} \frac{\delta E_{B}}{E_{B}}
$$

and thus $D$ can be determined with a relative precision of order $25 \%$ by any detector with reasonable statistics.

Finally, it is likely that the next supernova will lie in the Galactic plane (including the bulge). However, in our opinion, the point of the neutrino measurement is to make an unbiased estimate of the supernova location, so we do not use this as a constraint. For concreteness, we assume that $D=10 \mathrm{kpc}$ (approximately the distance to the Galactic center), but in an arbitrary direction.

\section{REACTIONS WITH ANGULAR DEPENDENCE}

Neutrinos are detected by their interaction with the target material, i.e., its electrons or nuclei. For some reactions, the angular distribution of the reaction products is correlated with the incoming neutrino direction. In this section we describe how this angular dependence can be used for determination of the supernova direction.

\section{A. Neutrino-electron scattering: Forward peaking}

Neutrino-electron scattering,

$$
\nu+e^{-} \rightarrow \nu+e^{-}
$$

occurs for all flavors of neutrinos and antineutrinos, and is detected by observing the recoil electrons with kinetic energy $T$ above the experimental threshold $T_{\min }$. The scattering angle is dictated by the kinematics and is given by

$$
\cos \alpha=\frac{E_{\nu}+m_{e}}{E_{\nu}}\left(\frac{T}{T+2 m_{e}}\right)^{1 / 2} .
$$

Both SK and SNO hope to have a threshold of order $T_{\min }$ $=5 \mathrm{MeV}$, and so $\cos \alpha \gtrsim 0.91$. However, after integrating over the electron kinetic energy distribution for a fixed neutrino energy, and also the neutrino energy spectrum, the average value will be larger. We take the latter to be of the Fermi-Dirac type with temperature $T=3.5,5$ and $8 \mathrm{MeV}$ for $\nu_{e}, \bar{\nu}_{e}$ and $\nu_{x} \equiv \nu_{\mu}, \bar{\nu}_{\mu}, \nu_{\tau}, \bar{\nu}_{\tau}$, respectively. Then we obtain $\langle\cos \alpha\rangle=0.98,0.97$, and 0.98 , respectively, with the combined average $\langle\cos \alpha\rangle=0.98$, corresponding to about $11^{\circ}$.

The angular distribution of the produced electrons is narrow, and depends on energy and flavor. However, multiple scattering of the electron will smear its Cerenkov cone. This washes out the dependence on energy and flavor, and one can reasonably model the electrons as having a Gaussian smearing from the forward direction, with a one-sigma width of $25^{\circ}$, for all energies and flavors. This is consistent with the estimate for SNO [16] and the measurement by a LINAC for SK [17]. The SK measurement shows that the angular resolution does depend on the electron energy, but the variation is not large.

Naively, if the one-sigma angular width of this cone is $\delta \alpha \simeq 25^{\circ}$, then the precision with which its center (i.e., the average) can be defined given $N_{S}$ events is

$$
\delta \theta \simeq \frac{\delta \alpha}{\sqrt{N_{S}}}
$$

where $\theta$ measures the angle from the best-fit direction (i.e., the average). For SK [14], $N_{S} \simeq 320$, so the cone center could be defined to within about $1.5^{\circ}$. For SNO (using both the light and heavy water) [15], $N_{S} \simeq 25$, so the cone center could be defined to about $5^{\circ}$. These results (at least for SK) are widely known (see, for example, Ref. [6]). The equivalent error on the cosine is $\delta(\cos \theta) \simeq(\delta \theta)^{2} / 2$, i.e., $3 \times 10^{-4}$ and $4 \times 10^{-3}$, respectively.

These results neglect the fact that the centroiding is to be done in two dimensions, and that this peak sits on a large background. It has been claimed that the error on centroiding in two dimensions is larger than the corresponding error in one dimension by a factor of $\sqrt{2}$. For $r=\sqrt{x^{2}+y^{2}}$, and uncorrelated errors of equal magnitude, $\delta x=\delta y=\sigma$, simple error propagation gives $\delta r=\sigma$. Only for correlated errors, e.g., a positive error $\delta x$ always accompanied by a positive $\delta y$ of the same magnitude, does the factor $\sqrt{2}$ appear. Centroiding in two dimensions is no harder than centroiding in one dimension since there are twice as many measurements, i.e., $x$ and $y$ for each point.

However, the nearly isotropic background from all other reactions, neglected in previous estimates, is more of a concern. Finding the supernova direction becomes a question of finding the centroid of a Gaussian peak of known width on a known flat background. The centroiding precision can be determined by a test due to Muller et al. [18]. This result follows from the assumption that a known template function with unknown centroid is adjusted until it gives the best least-squares fit to the data, i.e., exactly what one would do in practice. This technique does not require any arbitrary cuts on which data are included in determining the centroid (which would introduce bias). The appropriate twodimensional generalization is

$$
\frac{1}{(\delta x)^{2}}=\int d y \int d x \frac{[\partial L(x, y) / \partial x]^{2}}{L(x, y)}
$$

where $L(x, y)=d^{2} N / d x d y$, the density of events. Note that since the error is the same in any direction, and the $x$ and $y$ directions are arbitrary, we have considered the case in which the centroiding error $\delta x$ is only in the $x$ direction. One can show that this $\delta x$ is exactly the shift in the centroid which would increase the total $\chi^{2}$ of the fit by 1 . (One can also use the Rao-Cramer theorem, introduced in a later section, to show that this error $\delta x$ is the minimum which can be achieved by any technique.) 


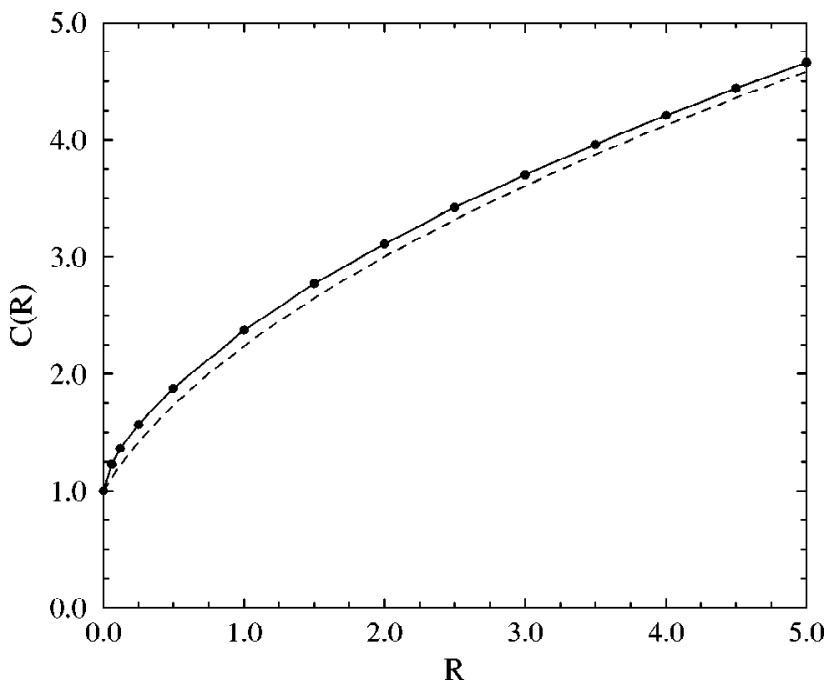

FIG. 1. The correction factor to the naive pointing error from $\nu+e^{-} \rightarrow \nu+e^{-}$scattering due to the isotropic background is shown. The solid line with points is the numerical result. The dashed line is $\sqrt{1+4 R}$, an approximation that is discussed in the text.

We use a two-dimensional Gaussian peak (with a total of $N_{S}$ signal events) on a flat background:

$$
L(x, y)=\frac{N_{S}}{2 \pi \sigma^{2}}\left[\exp \left(-\frac{x^{2}}{2 \sigma^{2}}\right) \exp \left(-\frac{y^{2}}{2 \sigma^{2}}\right)+R\right]
$$

where $R$ is the ratio of the heights of the flat background and the signal (at peak). For $N_{B}$ background events on the whole sphere,

$$
R=\frac{\sigma^{2}}{2} \frac{N_{B}}{N_{S}}
$$

where $\sigma=\delta \alpha \simeq 25^{\circ}$. Once $R$ has been specified, we treat the problem in a plane instead of on the sphere, since $\sigma$ is small. When $R=0$, the integral can be done analytically, with the expected result of $\delta x=\sigma / \sqrt{N_{S}}$. For $R>0$, it must be done numerically. We define a correction factor $C(R)$ to the naive error by

$$
C(R)=\frac{\delta x}{\sigma / \sqrt{N_{S}}},
$$

where the full error $\delta x$ is determined numerically from Eq. (6). The function $C(R)$ is shown in Fig. 1. Note that $C(R)$ depends on $R$ alone, so that the same figure can be used for both SK and SNO. Empirically, $C(R)$ is reasonably fit by $C(R) \simeq \sqrt{1+4 R}$. This form is motivated by two constraints: that $C(0)=1$, and that for $R \gg 1$, one can show from Eq. (6) that $C(R) \simeq \sqrt{4 R}$.

For SK and SNO, the number of signal events, $N_{S}$, is 320 and 25 , respectively. As a worst case, we assume that all events from other reactions are background events. Then for SK, $R \simeq 3.0$ and $C(R) \simeq 3.7$, and for $\mathrm{SNO}, R \simeq 3.8$ and $C(R) \simeq 4$.1. These correction factors may seem surprisingly large, but one should note that a two-sigma region contains about 2000 and 200 background events for SK and SNO, respectively. With the most pessimistic background assumptions, the centroiding errors for SK and SNO are then about $5^{\circ}$ and $20^{\circ}$, respectively.

It should be possible to reduce this isotropic background. In neutrino-electron scattering, the outgoing electrons tend to have energies well below the neutrino energy. In contrast, in the reaction $\bar{\nu}_{e}+p \rightarrow e^{+}+n$, the outgoing positron carries almost all of the neutrino energy. Approximately $2 / 3$ of these background events are above $20 \mathrm{MeV}$, and can be cut with little loss in signal. The background in the SNO heavy water will depend on the neutron detection technique. Crude estimates indicate that SK and SNO may each be able to attain $C(R) \simeq 2-3$.

\section{B. Neutrino-nucleus reactions: Weak angular dependence}

In this subsection we consider charged-current reactions on nuclear targets, i.e., reactions in which only $\nu_{e}$ and $\bar{\nu}_{e}$ participate. The reaction with the most events is $\bar{\nu}_{e}+p$ $\rightarrow e^{+}+n$, with $\simeq 10^{4}$ events expected in $\mathrm{SK}$ and $\simeq 400$ events expected in the light water of SNO. The other relevant reactions are those on deuterons in $\mathrm{SNO}$, with $\simeq 80$ events each expected for $\bar{\nu}_{e}+d \rightarrow e^{+}+n+n$ and $\nu_{e}+d \rightarrow e^{-}+p$ $+p$ [15]. We neglect charged-current reactions on the isotopes of oxygen [19], which also have weak asymmetries, but are difficult to separate from more dominant reactions. The reactions considered have lepton angular distributions approximately of the form

$$
\frac{d N}{d \cos \alpha}=\frac{N}{2}(1+a \cos \alpha),
$$

with, in general, $a=a\left(E_{\nu}\right)$. We will discuss the magnitude and variation of the coefficient $a$ shortly. We first consider how well one could localize the supernova assuming that $a$ is known and constant. In the following, we neglect the experimental angular resolution for electron and positron directions, as it will be negligible in comparison to the pointing errors discussed below.

Given a sample of events, one can attempt to find the axis defined by the neutrino direction. Along this axis, the distribution should be flat in the azimuthal angle $\phi$ and should have the form of Eq. (10) in $\cos \alpha$. Along any other axis, the distribution will be a complicated function of both the altitude and azimuthal angles. We assume that the axis has been found numerically, and ask how well the statistics allow the axis to be defined. A convenient way to assess that is to define the forward-backward asymmetry as

$$
A_{F B}=\frac{N_{F}-N_{B}}{N_{F}+N_{B}},
$$

where $N_{F}$ and $N_{B}$ are the numbers of events in the forward and backward hemispheres. The total number of events is $N=N_{F}+N_{B}$. Note that $A_{F B}$ will assume an extremal value $A_{F B}^{e x t r}$ along the correct neutrino direction. 

is

The error in $A_{F B}$ due to the statistical errors on $N_{F}$ and $N_{B}$

$$
\delta A_{F B}=\frac{1-A_{F B}^{2}}{2} \sqrt{\frac{1}{N_{F}}+\frac{1}{N_{B}}} .
$$

Using Eq. (10) one finds, simply,

$$
N_{F, B}=\frac{N}{2}\left(1 \pm \frac{a}{2}\right) \text {. }
$$

Therefore

$$
A_{F B}=\frac{a}{2}
$$

and

$$
\delta A_{F B}=\frac{1}{\sqrt{N}} \sqrt{1-\left(\frac{a}{2}\right)^{2}} \simeq \frac{1}{\sqrt{N}},
$$

where the error is nearly independent of $a$ for small $|a|$, which is the case for the reactions under consideration.

In the above, the coordinate system axis was considered to be correctly aligned with the neutrino direction. Now consider what would happen if the coordinate system were misaligned. While in general all three Euler angles would be needed to specify an arbitrary change in the coordinate system, symmetry considerations dictate that the computed value of $A_{F B}$ depends only upon one - the angle $\theta$ between the true and the supposed neutrino axis. Thus $A_{F B}$ is some function of $\theta$ if the axis is misaligned. Using a Legendre expansion, one can show that

$$
A_{F B}(\theta)=\frac{a}{2} \cos \theta
$$

The same expression is obtained by expressing the angular distribution in spherical harmonics and considering its behavior when acted on by the rotation operator. The error on the alignment is then

$$
\delta(\cos \theta)=\frac{2}{|a|} \delta A_{F B} \simeq \frac{2}{|a|} \frac{1}{\sqrt{N}} .
$$

As noted, one would find the best estimate of the neutrino axis numerically and define that direction to be $\cos \theta=1$. Along that axis, the measured asymmetry will be $A_{F B}^{\text {extr }}$. The above considerations describe the situation when the fluctuations do not dominate the value of $A_{F B}^{\text {extr }} \simeq a / 2 \pm 1 / \sqrt{N}$. Only in that case can one hope to use the angular distribution for pointing, and at the same time avoid apparent formal difficulties such as an infinite error in the cosine when $a \rightarrow 0$ or a possibility of $|\cos \theta|>1$ in Eq. (16).

Treating the nucleons as infinitely heavy, the coefficient $a$ in Eq. (10) is related to the competition of the Fermi (no spin flip) and Gamow-Teller (spin flip) parts of the matrix element squared:

$$
a=\frac{\left|M_{F}\right|^{2}-\left|M_{G T}\right|^{2}}{\left|M_{F}\right|^{2}+3\left|M_{G T}\right|^{2}} .
$$

For the $\bar{\nu}_{e}+p \rightarrow e^{+}+n$ reaction, $\left|M_{G T} / M_{F}\right|=1.26$ and thus $a \simeq-0.1$. However, as we have shown elsewhere [20], due to recoil and weak magnetism corrections of order $1 / M_{p}$, where $M_{p}$ is the proton mass, the coefficient $a$ varies quite rapidly with neutrino energy and changes sign near $E_{\nu}$ $=15 \mathrm{MeV}$, becoming positive at higher energies (see also Ref. [21]). In fact, after averaging over the $\bar{\nu}_{e}$ spectrum, which is taken as before as being a Fermi-Dirac distribution with a temperature $T=5 \mathrm{MeV}$, we obtain $\langle a\rangle \simeq+0.08$.

As stated above, for $\bar{\nu}_{e}+p \rightarrow e^{+}+n$ one expects $N \simeq 10^{4}$ events in SK. This would imply $\delta(\cos \theta) \simeq 0.2$ if $a=-0.1$ and a similar error if $a=+0.08$ and the temperature is known to be $T=5 \mathrm{MeV}$ (otherwise an uncertainty in the temperature would obviously cause an additional uncertainty in $\cos \theta$ ). The temperature can be measured from the shape of the positron spectrum, with a precision of the order of $1 \%$ $[14,15]$. Thus, even though the asymmetry parameter $a$ is quite small, the number of events is large enough that this technique in SK could give a reasonable pointing error. There are also events of this type in the light water of SNO. However, $N \simeq 400$, so this would imply $\delta(\cos \theta) \simeq 1.0$, which is too large to be useful.

The reactions on deuterons in the $\left(M_{p} \rightarrow \infty\right)$ approximation are pure Gamow-Teller transitions and thus $a=-1 / 3$. We will, for the sake of an estimate, assume that for the reactions on deuterons the energy dependence of $a$ can be neglected (though see Ref. [22]). We assume optimistically that the signal in SNO of the reactions $\bar{\nu}_{e}+d \rightarrow e^{+}+n+n$ and $\nu_{e}+d \rightarrow e^{-}+p+p$ can be combined, so $N \simeq 160$. With $a=-1 / 3$ this gives $\delta(\cos \theta) \simeq 0.5$, which is again rather large.

In the reaction $\bar{\nu}_{e}+p \rightarrow e^{+}+n$, the kinematics dictate that the outgoing neutrons have a forward angular distribution. If the positions of the positrons and the neutrons can be separately determined, the vector between these points can give some information on the neutrino direction, at least on a statistical basis [23]. In fact, this effect was observed in the Goesgen [24] and Chooz [25] reactor experiments. It is not currently possible to detect neutrons in SK or the light water in SNO, and we do not consider this further. However, this technique may allow a scintillator detector to have some pointing ability [20].

The techniques of this subsection will not allow the supernova to be located anywhere near as precisely as by neutrino-electron scattering. Nevertheless, they may provide an independent confirmation of the neutrino-electron scattering results, which would increase the confidence that a real supernova was seen. For example, consider the positrons from $\bar{\nu}_{e}+p \rightarrow e^{+}+n$ in SK. Along the axis determined by neutrino-electron scattering, the measured value should be $A_{F B} \simeq+0.04$, with an error of 0.01 , a four-sigma effect. This can probably be improved somewhat by considering only the highest-energy positrons. While the number of events will be reduced, the average $a$ will be increased [20], thus improving the pointing ability. 


\section{TRIANGULATION}

For two detectors separated by a distance $d$, there will be a delay between the arrival times of the neutrino pulse. The magnitude of the delay $\Delta t$ depends upon the angle $\theta$ between the supernova direction and the axis connecting the two detectors. Given a measured time delay $\Delta t$, the unknown angle $\theta$ can be determined:

$$
\cos \theta=\frac{\Delta t}{d}
$$

The Earth diameter is $d \approx 40 \mathrm{~ms}$. For a typical pair of detectors, the time will be somewhat less; for SK and SNO, $d$ $\approx 30 \mathrm{~ms}$. The error in the time delay will cause an error in the determination of $\theta$ :

$$
\delta(\cos \theta)=\frac{\delta(\Delta t)}{d}
$$

Thus two detectors define a cone along their axis with opening $\cos \theta$ and thickness $2 \times \delta(\cos \theta)$ in which the supernova can lie. Obviously, in order to have a reasonable pointing accuracy from triangulation, one will need $\delta(\Delta t) \ll d$. In what follows we discuss whether an appropriate time delay can be defined and what its error would likely be.

Note that this simple error analysis would have to be modified near $\Delta t= \pm d$, since we must have $|\cos \theta| \leqslant 1$, but we ignore this complication. Also, for convenience we use $\cos \theta$ rather than $\theta$ itself. Naively, $\delta \theta=\delta(\cos \theta) / \sin \theta$. This is indeed valid for moderate angles, but has spurious singularities at $\theta=0, \pi$. In fact, for small $\delta \theta$, one has $\delta \theta$ $\simeq \sqrt{2 \delta(\cos \theta)}$ near $\theta=0, \pi$.

For now we will consider just two detectors, SK and SNO, taking events from all reactions in SK and the light water in SNO. These are about $10^{4}$ and 400 events, respectively, mostly $\bar{\nu}_{e}+p \rightarrow e^{+}+n$. The effect of multiple detectors will be discussed later. Further, we make the following assumptions: that the detectors have perfect efficiency at all energies, perfect time resolution and synchronization, no dead time, and a negligible time-independent background. In practice, these should be reasonable assumptions. Therefore, we consider that SK and the light water in SNO are ideal detectors, identical except for size. The event rates in the two detectors should then be identical, except for normalization, fluctuations, and a delay $\Delta t$. That is, we are considering the best that triangulation could do under any circumstances, limited only by statistical errors.

The supernova neutrino pulse and hence also the observable scattering rate are assumed to have a short rise, followed by a relatively slow decline. The total duration of the rise time is unknown, but in many supernova models it is of the order of $100 \mathrm{~ms}$ [26]. The total duration of the decaying phase is much longer, and in most supernova models is a few seconds [26]. However, the observed duration of SN 1987A was clearly longer, about $10 \mathrm{~s}$, and we will base our estimates on that.

If we wish to assume as little as possible about the form of the event rate, we could simply consider the shift in the

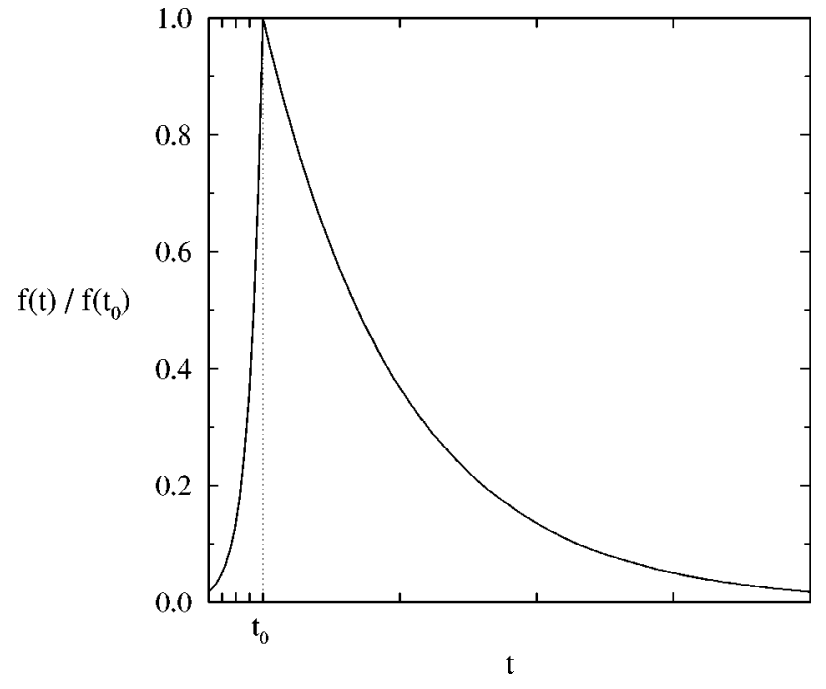

FIG. 2. The schematic form of the normalized event rate $f(t)$ is shown. To the left of $t_{0}$ there is an exponential rise with time constant $\tau_{1}$. To the right of $t_{0}$ there is an exponential decay with time constant $\tau_{2}$. The tick marks on the $t$ axis are in units of the respective time constants $\tau_{1}$ and $\tau_{2}$. For clarity of display, we used $\tau_{1} / \tau_{2} \simeq 10^{-1}$ in the figure, instead of the $\tau_{1} / \tau_{2} \simeq 10^{-2}$ assumed in the analysis.

average arrival time $\langle t\rangle$ between the two detectors. The expected value is just $\Delta t$. The error on the determination of the average arrival time is the width of the pulse divided by the square root of the number of events. Quite generally, this must be of order $3 \mathrm{~s} / \sqrt{10^{4}} \simeq 30 \mathrm{~ms}$ for $\mathrm{SK}$ and $3 / \sqrt{400}$ $\simeq 150 \mathrm{~ms}$ for $\mathrm{SNO}$, and therefore not useful for triangulation. One can show that a Kolmogorov-Smirnov test for a delay between the SK and SNO data leads to the same result for the error.

In our previous studies of the effect of a $\nu_{\tau}$ mass on the neutral-current event rate in SK or SNO [14,15], we compared the average arrival time from the neutral-current events to the average arrival time from the charged-current events. We showed that this allows the detection of a delay and the extraction of a $\nu_{\tau}$ mass with only minimal assumptions about the time dependences of the event rates. The results obtained demonstrated the best that one could do without assuming specific models for the event rates.

Evidently, the triangulation problem will require more detailed assumptions about the time dependence of the event rate. As noted above, here we have an additional piece of information: that besides the delay $\Delta t$, the event rates in SK and the light water in SNO should differ only in normalization and fluctuations. As a schematic model, we consider an event rate which consists of an exponential rise with a short time scale $\left(\tau_{1} \simeq 30 \mathrm{~ms}\right.$ ), followed by slower exponential decay $\left(\tau_{2} \simeq 3 \mathrm{~s}\right)$. The point of transition between the two is labeled $t_{0}$. In Fig. 2, the normalized event rate $f(t)$ is shown. We show below how the results depend upon the time scales $\tau_{1}$ and $\tau_{2}$. Since SK will have many more events than SNO, one could effectively measure the event rate in SK and use that to replace our assumed form. 


\section{A. Zero rise time case}

We consider first an even simpler model, in which the rise time $\tau_{1}=0$. Then the normalized event rate can be written

$$
f(t)=\frac{1}{\tau_{2}} \exp \left[-\frac{\left(t-t_{0}\right)}{\tau_{2}}\right], \quad t>t_{0},
$$

and is zero otherwise. If $f(t)$ is guaranteed to have an infinitely sharp edge, as above, one can show (see Refs. [2729]) that the best estimator (i.e., the technique with the smallest error) of the edge $t_{0}$ is

$$
t_{0} \simeq t_{1}-\frac{\tau_{2}}{N}
$$

where $t_{1}$ is the measured time of the first event. The error on the determination of $t_{0}$ is

$$
\delta\left(t_{0}\right)=\frac{\tau_{2}}{N}
$$

Here $\tau_{2} / N$ is simply the spacing between events near the peak. For a more general $f(t)$, but still with a sharp edge at $t_{0}$, one would simply replace $\tau_{2}$ by $1 / f\left(t_{0}\right)$. In fact, the shape of $f(t)$ is irrelevant except for its effect on the peak rate, i.e., $f\left(t_{0}\right)$. As long as $f(t)$ has a sharp edge and the right total duration, allowing a more general time dependence would therefore not change the results significantly. That is, only the long time scale $\tau_{2}$ is important, since it determines the event spacing near $t_{0}$.

The event rate in SNO will consist of $N$ events sampled from $f\left(t, t_{0}\right)$, and the event rate in SK will consist of $N^{\prime}$ events sampled from $f\left(t, t_{0}^{\prime}\right.$ ) (where here we show the offset explicitly). The parameters $t_{0}$ and $t_{0}^{\prime}$ can be extracted from the times of the first events as above. Then $\Delta t=t_{0}-t_{0}^{\prime}$, and its error will be dominated by the error in $t_{0}$ as extracted at $\mathrm{SNO}$, so that $\delta(\Delta t) \simeq \delta t_{0}$. For $\tau_{2}=3 \mathrm{~s}$, this idealized model would allow the offset of the edge to be measured to $\simeq 0.3 \mathrm{~ms}$ in $\mathrm{SK}$ and $\simeq 8 \mathrm{~ms}$ in SNO. This gives $\delta(\cos \theta)$ $\simeq 0.25$ at one sigma.

This technique of using the first event only works if there is no time-independent background and if there is absolutely no tail of $f(t)$ before $t_{0}$. In either case, the fluctuation of a single unwanted event can change the extracted delay in an unpredictable way. At the cost of an increase in error, this technique could be made robust by looking at the average time of the first few events. In all of the other techniques discussed in this paper, the role of the time-independent background is negligible.

\section{B. Nonzero rise time case}

The above model has somewhat limited use, since the assumption of a zero rise time does not seem to be justified. As noted, the supernova models suggest a nonzero rise time, of the order of $\tau_{1} \simeq 30 \mathrm{~ms}$, related to the shock propagation time across the supernova core. If the rise time is nonzero, the results of the previous subsection cannot be used. The error in $t_{0}$ is only given by the spacing between events at the peak if the edge is sharp. In this subsection, we allow a nonzero rise time and show that the results have a qualitatively different dependence on the parameters than in the previous case. In addition, softening the leading edge will obviously make the triangulation error larger.

For the normalized event rate, we take

$$
\begin{array}{ll}
f(t)=\alpha_{1} \times \frac{1}{\tau_{1}} \exp \left[+\frac{\left(t-t_{0}\right)}{\tau_{1}}\right], & t<t_{0}, \\
f(t)=\alpha_{2} \times \frac{1}{\tau_{2}} \exp \left[-\frac{\left(t-t_{0}\right)}{\tau_{2}}\right], & t>t_{0},
\end{array}
$$

where

$$
\alpha_{1}=\frac{\tau_{1}}{\tau_{1}+\tau_{2}}, \quad \alpha_{2}=\frac{\tau_{2}}{\tau_{1}+\tau_{2}}
$$

Then $f(t)$ is a normalized probability density function built out of two exponentials and joined continuously at $t=t_{0}$. In what follows, we assume that this form of $f(t)$ is known to be correct and that $\tau_{1}$ and $\tau_{2}$ are known.

As above, the event rate in SNO will consist of $N$ events sampled from $f\left(t, t_{0}\right)$, and the event rate in SK will consist of $N^{\prime}$ events sampled from $f\left(t, t_{0}^{\prime}\right)$. Then $\Delta t=t_{0}-t_{0}^{\prime}$, and again $\delta(\Delta t) \simeq \delta t_{0}$, since the SNO error dominates. We consider only the statistical error determined by the number of counts. Any uncertainties in the form of $f(t)$ or its parameters will only increase the error. As noted, we want to determine the minimal error on the triangulation.

This model, while simple, contains the essential time scales and an adjustable offset. More general models for the event rate must reproduce these time scales in order to be physically plausible, and so the final value for the error would be close to what is obtained here. To define the time delay between the two pulses, we have used the offset at which the rising and falling exponentials are joined. However, in the final result for the error in the delay, the particular way in which the offset time is defined drops out and the result is therefore quite general. That is, this simple model for the event rate is general enough for calculating the statistical error in the measured delay.

These considerations lead to a well-posed statistical problem: If $N$ events are sampled from a known distribution $f\left(t, t_{0}\right)$, how well can $t_{0}$ be determined? The Rao-Cramer theorem $[27,30]$ provides an answer to this question. This theorem allows one to calculate the minimum possible variance in the determination of a parameter (here $t_{0}$ ) by any technique whatsoever. This minimum variance can be achieved when all of the data are used as "efficiently" as possible, which is frequently possible in practice. One requirement of the theorem is that the domain of positive probability must be independent of the parameter to be determined. This condition is obviously not met for a zero rise time, since then the domain is $\left(t_{0}, \infty\right)$. For a nonzero rise time, the domain is technically $(-\infty, \infty)$, independent of $t_{0}$, and so the theorem applies. The minimum possible variance in the determination of $t_{0}$ is 


$$
\frac{1}{\left(\delta t_{0}\right)_{\min }^{2}}=N \int d t f\left(t, t_{0}\right)\left[\frac{\partial \ln f\left(t, t_{0}\right)}{\partial t_{0}}\right]^{2} .
$$

This is the general form for an arbitrary parameter $t_{0}$. When $t_{0}$ is a translation parameter, i.e., when $f\left(t, t_{0}\right)$ depends only on $t-t_{0}$, this reduces to

$$
\begin{aligned}
\frac{1}{\left(\delta t_{0}\right)_{\min }^{2}} & =N \int d t f\left(t, t_{0}\right)\left[\frac{\partial \ln f\left(t, t_{0}\right)}{\partial t}\right]^{2} \\
& =N \int d t \frac{\left[\partial f\left(t, t_{0}\right) / \partial t\right]^{2}}{f\left(t, t_{0}\right)} .
\end{aligned}
$$

The latter form was independently derived by another method in Ref. [18]. For the particular choice of $f\left(t, t_{0}\right)$ above, this reduces to

$$
\frac{1}{\left(\delta t_{0}\right)_{\min }^{2}}=N\left(\alpha_{1} / \tau_{1}^{2}+\alpha_{2} / \tau_{2}^{2}\right) .
$$

For $\tau_{1} \ll \tau_{2}$, the minimum error is then

$$
\left(\delta t_{0}\right)_{\min } \simeq \frac{\sqrt{\tau_{1} \tau_{2}}}{\sqrt{N}} \simeq \frac{\tau_{1}}{\sqrt{N_{1}}} .
$$

Note that $N_{1} \simeq N\left(\tau_{1} / \tau_{2}\right)$ is the the number of events in the rising part of the pulse. Since the rise is the sharpest feature in $f(t)$, it is unsurprising that it contains almost all of the information about $t_{0}$. The total number of events, $N$, is fixed by the supernova binding energy release. A change in the total duration of the pulse, i.e., $\tau_{2}$, would therefore affect the peak event rate and hence the fraction of events in the leading edge, i.e., $N_{1} / N=\alpha_{1} \simeq \tau_{1} / \tau_{2}$. That is, for $N$ fixed, we are considering how a change in the assumed value of $\tau_{2}$ would affect the timing sensitivity; note that $\tau_{2}$ appears only via the fraction of events in the leading part of the pulse. For a more general $f(t)$, one would replace $\tau_{1} / \tau_{2}$ by this fraction computed directly.

For $\mathrm{SNO}, \quad N_{1} \simeq 10^{-2} \times 400 \simeq 4$, so $\delta\left(t_{0}\right) \simeq 30 \mathrm{~ms} / \sqrt{4}$ $\simeq 15 \mathrm{~ms}$. Since SK has about 25 times more events, the corresponding error would be about $3 \mathrm{~ms}$. Therefore, the error on the delay is $\delta(\Delta t) \simeq 15 \mathrm{~ms}$ and $\delta(\cos \theta) \simeq 0.50$ at one sigma. We have not yet specified the method for extracting $t_{0}$ and hence $\Delta t$ from the data. That is exactly the point of the Rao-Cramer theorem - that one can determine the minimum possible error without having to try all possible methods. A possible technique which should come close to achieving this minimal error is discussed below.

For the two cases, zero and nonzero rise time, we used different mathematical techniques which were applicable only in one case or the other. This may seem like an artificial distinction and that these two cases do not naturally limit to each other. In particular, it may seem incompatible that in the first case the error $\sim 1 / N$, while in the second the error $\sim 1 / \sqrt{N}$. Further, one obviously cannot take $\tau_{1} \rightarrow 0$ in the results of this subsection.
However, there is physically a natural joining of the two results. For the case of $\tau_{1}=0$, we found that $\delta\left(t_{0}\right) \simeq 8 \mathrm{~ms}$ for SNO. For the case of $\tau_{1}>0$, we chose $\tau_{1}=30 \mathrm{~ms}$ and found $N_{1} \simeq 4$ and $\delta\left(t_{0}\right) \simeq 15 \mathrm{~ms}$ for SNO. If we reduce $\tau_{1}$ by a factor 4 , so that $\tau_{1}=7.5 \mathrm{~ms}$, then this error is reduced by a factor 2 so that $\delta\left(t_{0}\right) \simeq 7.5 \mathrm{~ms}$. But now there is only $N_{1}$ $\simeq 1$ event in the rising part of the pulse. At this point, the difference between $\tau_{1}=0$ and $\tau_{1} \leqslant 7.5 \mathrm{~ms}$ becomes difficult to distinguish; i.e., the edge appears sharp. That is, the two techniques give the same numerical result for the error at the boundary between the two cases.

\section{What will the event rate really look like?}

We considered two simple models for what the event rates might look like. Those models were of course crude, yet they illustrate how the different time scales affect the final results. The short rise $\left(\sim \tau_{1}\right)$ at the beginning of the pulse is a prominent feature, and it provides most of the timing information. The long decay $\left(\sim \tau_{2}\right)$ is important principally through how it affects the normalization, i.e., the number of events before or near the maximum.

The rise time, set by the time scale $\tau_{1}$, may be smaller than suggested in Ref. [26]. For example, in some of the models considered in Ref. [31], the duration of the rise does appear to be shorter than considered here. In some cases, the $\bar{\nu}_{e}$ luminosity rises nearly instantaneously to a given value and then more slowly to a peak value which is several times larger. In this case, a zero-rise-time analysis may be appropriate, and the error in $t_{0}$ depends on the spacing between events. However, one must not use the peak event rate, but rather the event rate at the point where the rise is no longer instantaneous. This will give an error several times larger than the zero-rise-time case used above, where the instantaneous portion rose all the way to the peak.

The neutrino pulse duration, set by the time scale $\tau_{2}$, may also be smaller than assumed here, and our results can easily be scaled appropriately. Note that in the zero-rise-time case, the error $\sim \tau_{2}$, and in the nonzero-rise-time case, the error is $\sim \sqrt{\tau_{2}}$. Thus in the latter case, even if $\tau_{2}=1 \mathrm{~s}$, the triangulation error would improve by only a factor of $\sqrt{3}$, and so $\delta(\cos \theta) \simeq 0.3$, still rather large. However, one has to keep in mind that our choice $\tau_{2}=3 \mathrm{~s}$ was motivated by the $\mathrm{SN}$ 1987A observation that $25 \%$ of the events arrived at least $5 \mathrm{~s}$ after the start of the pulse. While the statistics were poor, a time scale of $\tau_{2}=1 \mathrm{~s}$ or smaller seems to be unlikely. It may also be that the decay of the neutrino pulse is characterized by two time scales - a quick drop, followed by a slower decline, e.g., a simplification of the gradually decreasing time scale considered in Ref. [32]. Even so, if the duration of SN 1987A is reproduced, there would be little change in the error in the delay.

The event rate may also have a much more complex structure than assumed here. For example, there could be oscillations or other sharp features which could be used to define a time from which to measure the delay. However, since the most prominent possible sharp feature, a zero-rise-time edge, is not enough for a successful triangulation, any such features should be less significant. Moreover, one should not 
TABLE I. One-sigma errors on how well the direction to the supernova is defined by various techniques, at $D=10 \mathrm{kpc}$. The other parameters used are noted in the text. For neutrino-electron scattering, the most pessimistic background assumptions were used.

\begin{tabular}{lc}
\hline \hline \multicolumn{1}{c}{ Technique } & Error \\
\hline$\nu+e^{-}$forward scattering (SK) & $\delta \theta \simeq 5^{\circ}, \delta(\cos \theta) \simeq 4 \times 10^{-3}$ \\
$\nu+e^{-}$forward scattering (SNO) & $\delta \theta \simeq 20^{\circ}, \delta(\cos \theta) \simeq 6 \times 10^{-2}$ \\
\hline $\bar{\nu}_{e}+p$ angular distribution (SK) & $\delta(\cos \theta) \simeq 0.2$ \\
$\bar{\nu}_{e}+p$ angular distribution (SNO) & $\delta(\cos \theta) \simeq 1.0$ \\
$\nu_{e}+d, \bar{\nu}_{e}+d$ angular distributions (SNO) & $\delta(\cos \theta) \simeq 0.5$ \\
\hline Triangulation (SK and SNO) & $\delta(\cos \theta) \simeq 0.5$ \\
\hline \hline
\end{tabular}

forget that the whole point of the supernova early alert network is to use an automated analysis to determine the direction from the data. For the result to be available essentially immediately, the analysis should assume as little as possible about the shape of the pulse. That was part of our motivation to consider a simple model characterized only by time scales which are reasonably well known.

Note also that we neglected events in the prompt burst of electron neutrinos. The expected number of events is very small (for example, Ref. [33] has $N \sim 1$ in SNO), although there is considerable variation in the predictions for the number of events and the duration of this burst. It seems unlikely that the timing error would be small enough to be useful or that the predictions are robust enough to allow an automated analysis.

In any case, the scenario for attempted triangulation could be the following: the detector with the largest statistics, e.g., $\mathrm{SK}$, could be used to determine the fraction $N_{1} / N$ of events in the leading edge and the duration of the rise time $\tau_{1}$ (both of these will have some errors, unlike what we assumed in using the Rao-Cramer theorem). This fraction would then specify which of the SNO events were to be considered as coming from the leading edge. The delay could then be determined by the time difference of the average arrival times of leading edge events in SK and SNO. The error would then be $\simeq \tau_{1} / \sqrt{N_{1}}$, where $N_{1}$ is the number of events in SNO coming from the rising edge. Thus this technique might approximately attain the Rao-Cramer lower bound on the error. Alternatively, if SK determines that the zero-rise-time model is applicable, the delay could be extracted from the time difference of first events in each detector. In this case, the error is again determined by SNO, and would be the event spacing near the peak.

\section{Comparison to other work}

In Ref. [10], the triangulation error for a zero-rise-time pulse using SK and SNO was also considered, with a final result of $1.3 \mathrm{~ms}$, to be compared with our result of $8 \mathrm{~ms}$. The difference is due to different input parameters. We assumed 400 events in the light water of SNO, an exponential decay of the event rate with $\tau_{2}=3 \mathrm{~s}$, and a distance of $D$ $=10 \mathrm{kpc}$. In Ref. [10], it was assumed that all flavors and all reactions could be combined (we argue against this below), for $10^{3}$ events in total (at a distance of $10 \mathrm{kpc}$ ), with half in the first $1 \mathrm{~s}$. The greater number of events and the shorter assumed duration of the pulse make the event rate at peak $500 \mathrm{~s}^{-1}$ instead of $133 \mathrm{~s}^{-1}$. In addition, the final error was scaled to a distance of $8 \mathrm{kpc}$. After correcting for these differences, the results are in agreement. The same holds for the results in Ref. [6], where again a peak rate of $500 \mathrm{~s}^{-1}$ for SNO and a zero rise time were assumed.

In Ref. [11], a different technique was proposed, which does not explicitly specify whether or not the rise time is nonzero. The proposed technique begins by constructing the cumulative distributions (this function increases by a step of $1 / N$ at each event time, and is discrete but not binned) for SK and SNO. At least for the light water events, these functions should be the same up to fluctuations and a possible delay. The proposal is to make a simple low-order fit to the beginning of each cumulative distribution and to extract the delay from the difference of the intercepts. A preliminary delay error of order $5 \mathrm{~ms}$ was presented (for $D=10 \mathrm{kpc}$ ). However, the fit to the cumulative distribution function does not yet take into account the fact that the errors on successive steps are highly correlated (because most of the data at a given step are the data from the previous step). Taking this into account will increase the error. In any case, the error from this proposed method cannot be smaller than that from the time of the first event (zero-rise-time case) or the RaoCramer result (nonzero-rise-time case), whichever is appropriate.

\section{CONCLUSIONS AND DISCUSSION}

The final uncertainties, calculated for a canonical supernova $10 \mathrm{kpc}$ away and with a total energy release of 3 $\times 10^{53}$ ergs, are summarized in Table I. These are all one sigma errors, though larger confidence regions may be necessary for making a search for a supernova.

Neutrino-electron scattering has the best pointing precision. Moreover, the calculated precision is largely independent of assumptions about the supernova model. In particular, it is totally independent of the time dependence of the event rate. The isotropic background from other reactions degrades the precision somewhat, but it is still the most precise technique.

The angular asymmetry of positrons from the $\bar{\nu}_{e}+p$ $\rightarrow e^{+}+n$ reaction, even when combined with the high statis- 
tics of SK, does not give a comparably small pointing error. It makes it possible, however, to check that the signal is indeed coming from the right direction. The angular distributions from the charged-current deuteron reactions are even weaker.

Under realistic assumptions about the numbers of events and the time scales, triangulation with SK and SNO appears to be very difficult, if not impossible. Other tests for the time delay can be considered. However, for either a zero rise time or a nonzero rise time, we have shown in the previous section what the smallest possible errors are. For fixed inputs, there is simply no way to do better.

But so far in this paper all of the concrete results were based on using just two detectors, SK and SNO, and taking all events in the light water (these are dominated by the charged-current signal $\left.\bar{\nu}_{e}+p \rightarrow e^{+}+n\right)$. Can the pointing accuracy, in particular for the triangulation technique, improve if other existing or planned detectors are used?

First, we stress again that for the method to succeed the signals in different detectors must differ only in the normalization, fluctuations, and a possible delay. For example, that precludes including the neutral current events (dominated by $\nu_{\mu}, \nu_{\tau}$, and their antiparticles) from the heavy water portion of SNO. That is because one cannot guarantee that the time dependence of the scattering rate for these events is the same as for the events in the light water. In fact, at the crucial early times, the supernova models suggest that there are differences among the flavors. Since the time dependences of the luminosities and temperatures are not known to the needed high precision, these differences cannot be corrected for.

There are several detectors, existing or under construction, that will observe a few to several hundred $\bar{\nu}_{e}+p \rightarrow e^{+}$ $+n$ events. In particular the existing MACRO and LVD detectors in Gran Sasso, and Borexino and KamLAND detectors under construction will be clearly able to "see" a Galactic supernova (see Refs. [34-37], respectively), and can undoubtedly contribute to the false signal elimination in the planned early alert network. However, since these detectors are based on scintillation rather than on Cerenkov light, it is not $a$ priori clear that the basic requirement of the similarity of response is indeed satisfied. And even if that difficulty could be overcome, the numbers of events in those detectors will be comparable to what is expected for SNO. Thus our estimate of the uncertainty associated with triangulation in the last line of Table I is valid for them also. With three detectors, there is in principle an improvement in the pointing from triangulation. However, that improvement is minimal if the pointing error from any two detectors is of order half of the sky.

The AMANDA detector (or its successor) can perhaps observe a supernova via the $\bar{\nu}_{e}+p \rightarrow e^{+}+n$ reaction in a very large target volume [38]. However, the principle used for supernova detection will be a fluctuation in the (large) background rate. Over an interval of a few seconds, the background events dominate the signal events, $N_{B} \gg N_{S}$, but $N_{S}$ $\gg \sqrt{N_{B}}$. The actual supernova events can only be distinguished in a statistical sense. Under these circumstances, it will not be possible to map out the event rate well enough to make a precise measurement of $t_{0}$ or some other appropriate time.

What would it take to make triangulation viable? As a simple example, we consider SK and a hypothetical second detector, also with $\simeq 10^{4}$ events at $10 \mathrm{kpc}$, and a separation of $30 \mathrm{~ms}$ between the two. The second detector might be very similar to SK, or it might be primarily sensitive to neutral-current reactions (in the latter case, we assume, despite the strong cautions above, that the charged-current and neutral-current event rates can be directly compared for timing purposes). For the event rate assumed in our main analysis, each detector would have a timing error of $\delta\left(t_{0}\right)$ $\simeq 3 \mathrm{~ms}$, so that the triangulation pointing error would be $\delta(\cos \theta) \simeq 0.15$. Results for the more general case of combining several detectors with different timing errors have been given in Refs. [2,10].

The pointing error from the angular distributions always scales with $1 / \sqrt{N}$. Under the assumption that the event rate rise time is nonzero [26], the triangulation pointing error also scales with $1 / \sqrt{N}$. Since $N \sim 1 / D^{2}$, all of the errors scale linearly with the distance $D$. The triangulation measurement may then become feasible if the distance to the supernova is significantly less than $10 \mathrm{kpc}$. However, all of the other techniques improve by the same factor. (However, if the rise time were vanishing [31], then the triangulation error would scale as $1 / N \sim D^{2}$; see the discussion above.)

Thus our analysis shows that a Galactic supernova can indeed be located by its neutrino signal and that, among the possible methods, the best technique by a large margin is neutrino-electron scattering in a water Cerenkov detector. Currently, either SK or SNO can separately make this measurement. In the above, we considered the directional information from the neutrino data alone. The operators of the alert network or astronomers themselves can of course combine these results with a Galactic model of where a supernova is likely to be. Our results indicating that triangulation will be very difficult do not mean that the data sharing among different detectors is not worthwhile. Only a coincidence of two or more detectors can eliminate false alarms and be the basis of a reliable early alert system.

\section{ACKNOWLEDGMENTS}

This work was supported in part by the U.S. Department of Energy under Grant No. DE-FG03-88ER-40397. J.F.B. was supported by Caltech. We thank Kate Scholberg, Alec Habig, Mark Vagins, Adam Burrows, and the other participants in the Supernova Early Alert Network Workshop for discussions on supernova location, and Robert Sherman and Brad Filippone for discussions on statistics. In addition, we thank Mark Vagins for bringing this problem to our attention. 
[1] A. Habig and K. Scholberg, "An Early Supernova Detection System" (unpublished).

[2] A. Habig, K. Scholberg, and M. Vagins, poster at the Neutrino '98 Conference, 1998.

[3] First International Workshop on the Supernova Early Alert Network, Boston University, 1998, and bound photocopied transparencies of the same.

[4] D.B. Cline, in Trends in Astroparticle Physics, edited by D. Cline and R. Peccei (World Scientific, Singapore, 1992), p. 590.

[5] R.A. Muller et al., Astrophys. J. Lett. 384, L9 (1992); S. van den Bergh, Comments. Astrophys. 17, 125 (1993); G.A. Tammann, W. Löffler, and A. Schröder, Astrophys. J. Suppl. 92, 487 (1994); F.X. Timmes, R. Diehl, and D.H. Hartmann, Astrophys. J. 479, 760 (1997).

[6] A. Burrows, D. Klein, and R. Gandhi, Phys. Rev. D 45, 3361 (1992).

[7] G.V. Domogatsky and G.T. Zatsepin, in Proceedings of the 9th International Conference on Cosmic Rays (Institute of Physics and the Physics Society, London, 1966), Vol. 2, p. 1030.

[8] A.A. Belyaev, Yu.S. Kopysov, O.G. Ryazhskaya, and G.T. Zatsepin, in Neutrinos-78, edited by E.C. Fowler (Purdue University, West Lafayette, IN, 1978), p. 871.

[9] J.M. LoSecco, Science 224, 56 (1984).

[10] M. Vagins, in First International Workshop on the Supernova Early Alert Network [3].

[11] C. Virtue, in First International Workshop on the Supernova Early Alert Network [3].

[12] J.F. Beacom, in First International Workshop on the Supernova Early Alert Network [3].

[13] W.D. Arnett, J.N. Bahcall, R.P. Kirshner, and S.E. Woosely, Annu. Rev. Astron. Astrophys. 27, 629 (1989); B. Jegerlehner, F. Neubig, and G. Raffelt, Phys. Rev. D 54, 1194 (1996); H.A. Bethe, Rev. Mod. Phys. 62, 801 (1990), and references therein.

[14] J.F. Beacom and P. Vogel, Phys. Rev. D 58, 053010 (1998).

[15] J.F. Beacom and P. Vogel, Phys. Rev. D 58, 093012 (1998).

[16] G.T. Ewan et al., Sudbury Neutrino Observatory Proposal, Report No. SNO-87-12, 1987.

[17] M. Nakahata et al., Nucl. Instrum. Methods Phys. Res. A 421, 113 (1999).

[18] D.E. Muller, H.C. Hoyt, D.J. Klein, and J.W.M. DuMond,
Phys. Rev. 88, 775 (1952); see also P.G. Hansen, Nucl. Instrum. Methods 154, 321 (1978); P.L. Lee, Nucl. Instrum. Methods Phys. Res. A 243, 605 (1986).

[19] W.C. Haxton, Phys. Rev. D 36, 2283 (1987); W.C. Haxton and R.G.H. Robertson, Phys. Rev. C 59, 515 (1999).

[20] P. Vogel and J.F. Beacom, Phys. Rev. D (to be published), hep-ph/9903554.

[21] D.H. Perkins, in Ninth Workshop on Grand Unification, edited by R. Barloutard (World Scientific, Singapore, 1988), p. 170.

[22] A.B. Dobrotsvetov and S.A. Fayans, Phys. At. Nucl. 56, 455 (1993).

[23] C. Bemporad, in Third International Workshop on Neutrino Telescopes, edited by M. Baldo Ceolin (Padua University, Padua, Italy, 1991).

[24] G. Zacek, Ph.D. thesis, Technical University of Munich, 1984.

[25] C. Bemporad, Neutrino '98 Conference [2].

[26] Burrows, Klein, and Gandhi [6]; M. Herant et al., Astrophys. J. 435, 339 (1994); A. Mezzacappa et al., ibid. 495, 911 (1998); T. Totani et al., ibid. 496, 216 (1998).

[27] R.V. Hogg and A.T. Craig, Introduction to Mathematical Statistics, 4th ed. (Macmillan, New York, 1978).

[28] T. Dalenius, J. R. Stat. Soc. A 128, 110 (1965).

[29] W.T. Eadie et al., Statistical Methods in Experimental Physics (American Elsevier, New York, 1971).

[30] A. Stuart and J.K. Ord, Kendall's Advanced Theory of Statistics, 5th ed. (Oxford University Press, New York, 1991), Vol. 2.

[31] E.S. Myra and A. Burrows, Astrophys. J. 364, 222 (1990); F.D. Swesty, J.M. Lattimer, and E.S. Myra, ibid. 425, 195 (1994).

[32] A. Burrows and J.M. Lattimer, Astrophys. J. Lett. 318, L63 (1987).

[33] F.K. Sutaria and A. Ray, Phys. Rev. Lett. 79, 1599 (1997).

[34] M. Ambrosio et al., Astropart. Phys. 8, 123 (1998).

[35] M. Aglietta et al., Report No. INFN/AE-97/33.

[36] L. Cadonati, F.P. Calaprice, and M.C. Chen, Bull. Am. Phys. Soc. 43, 1563 (1998).

[37] P. Alivisatos et al., KamLAND Proposal, Report No. TOHOKU-RCNS-9815.

[38] F. Halzen, J.E. Jacobsen, and E. Zas, Phys. Rev. D 53, 7359 (1996). 